\title{
AGGREGATION OF A RANDOM-COEFFICIENT AR(1) PROCESS WITH INFINITE VARIANCE AND IDIOSYNCRATIC INNOVATIONS
}

\author{
DONATA PUPLINSKAITE,${ }^{*}$ Vilnius University and \\ Institute of Mathematics and Informatics, Vilnius \\ DONATAS SURGAILIS,** Institute of Mathematics and Informatics, Vilnius
}

\begin{abstract}
Contemporaneous aggregation of $N$ independent copies of a random-coefficient AR(1) process with random coefficient $a \in(-1,1)$ and independent and identically distributed innovations belonging to the domain of attraction of an $\alpha$-stable law $(0<\alpha<2)$ is discussed. We show that, under the normalization $N^{1 / \alpha}$, the limit aggregate exists, in the sense of weak convergence of finite-dimensional distributions, and is a mixed stable moving average as studied in Surgailis, Rosiński, Mandrekar and Cambanis (1993). We focus on the case where the slope coefficient $a$ has probability density vanishing regularly at $a=1$ with exponent $b \in(0, \alpha-1)$ for $\alpha \in(1,2)$. We show that in this case, the limit aggregate $\left\{\bar{X}_{t}\right\}$ exhibits long memory. In particular, for $\left\{\bar{X}_{t}\right\}$, we investigate the decay of the codifference, the limit of partial sums, and the long-range dependence (sample Allen variance) property of Heyde and Yang (1997).
\end{abstract}

Keywords: Aggregation; random-coefficient AR(1) process; infinite variance; mixed stable moving average; codifference; self-similar process; long memory

2010 Mathematics Subject Classification: Primary 62M10

Secondary 60G18; 60G52

\section{Introduction}

It is well known that heavy-tailedness and long memory are among the most important empirical 'stylized facts' of financial time series. See, e.g. Mikosch (2003) and the references therein. Since economic reasons for long memory in financial data are not very clear, attempts have been made to explain it using simple heterogeneous dynamic models involving regime shifts or aggregation. One of the most successful approaches in this direction, originating in Robinson (1978) and Granger (1980), is based on contemporaneous aggregation of heterogeneous random-coefficient $\mathrm{AR}(1)$ processes near the nonstationarity regime. Further justification of the aggregation procedure in linear models was provided in Gonçalves and Gouriéroux (1988), Oppenheim and Viano (2004), Zaffaroni (2004), and Celov et al. (2007). Contemporaneous aggregation of heterogeneous heteroscedastic models was discussed in Ding and Granger (1996), Leipus and Viano (2002), Kazakevičius et al. (2004), Zaffaroni (2007a), (2007b), and Giraitis et al. (2010).

Almost all of the above-mentioned papers refer to the case of finite-variance processes. Because heavy tails are important in financial modeling, aggregation of infinite-variance

Received 5 October 2009; revision received 20 March 2010.

* Postal address: Faculty of Mathematics and Informatics, Vilnius University, Naugarduko 24, LT-03225 Vilnius,

Lithuania. Email address: donata.puplinskaite@mif.stud.vu.lt

** Postal address: Institute of Mathematics and Informatics, Akademijos 4, LT-08663 Vilnius, Lithuania.

Email address: sdonatas@ktl.mii.lt 
dynamic models also presents considerable interest. Puplinskaite and Surgailis (2009) discussed contemporaneous aggregation of heterogeneous random-coefficient AR(1) models with common innovations in the domain of attraction of an $\alpha$-stable law, $0<\alpha<2$, and longmemory properties of the limiting aggregated process. They showed that in such cases, the limit aggregated process is a moving average with independent and identically distributed (i.i.d.) innovations, whose coefficients may depend hyperbolically as $j^{d-1}$ with the lag, for $0<d<1-1 / \alpha, 1<\alpha<2$. Let us note that the above aggregation scheme with a particular choice of beta-distributed slope coefficient leads to a $\operatorname{FARIMA}(0, d, 0)$ process with $\alpha$-stable innovations (see Puplinskaite and Surgailis (2009)).

In the present paper we discuss contemporaneous aggregation of infinite-variance heterogeneous $\mathrm{AR}(1)$ processes with idiosyncratic innovations (in other words, aggregation of independent copies of random-coefficient $\mathrm{AR}(1)$ processes). We show that, under some natural assumptions on the $\mathrm{AR}(1)$ noise and distribution of the slope coefficient, the limit aggregated process exists and is a so-called mixed stable moving average given in (1.3), below. The class of mixed stable moving average processes, introduced in Surgailis et al. (1993) extends (usual) $\alpha$-stable moving average processes, and plays an important role in the general theory of stationary $\alpha$-stable processes (see Rosiński (1995)).

Let us describe the main results of this paper. Let $\left\{X_{t}, t \in \mathbb{Z}\right\}$ be a stationary solution of the $\operatorname{AR}(1)$ equation

$$
X_{t}=a X_{t-1}+\varepsilon_{t},
$$

where $\left\{\varepsilon_{t}, t \in \mathbb{Z}\right\}$ are i.i.d. random variables (RVs) in the domain of the (normal) attraction of an $\alpha$-stable law, $0<\alpha<2$, and where $a$ is an RV, independent of $\left\{\varepsilon_{t}\right\}$ and satisfying $|a|<1$ almost surely (a.s.). Let the $X_{i t}=a_{i} X_{i, t-1}+\varepsilon_{i t}, i=1,2, \ldots$, be independent copies of (1.1). If the distribution of $a$ satisfies the condition that, for some $p<\alpha$,

$$
\mathrm{E}\left(1-|a|^{p}\right)^{-1}<\infty
$$

then

$$
N^{-1 / \alpha} \sum_{i=1}^{N} X_{i t} \stackrel{\text { FDD }}{\rightarrow} \bar{X}_{t},
$$

in the sense of weak convergence of finite-dimensional distributions, where the limit process is written as the stochastic integral

$$
\bar{X}_{t}=\sum_{s \leq t} \int_{(-1,1)} a^{t-s} M_{s}(\mathrm{~d} a),
$$

where $\left\{M_{s}, s \in \mathbb{Z}\right\}$ are i.i.d. copies of an $\alpha$-stable random measure $M$ on $(-1,1)$ with control measure proportional to the distribution $\Phi$ of the RV $a$ (Theorem 2.1). Below, we call $\Phi$ the mixing distribution of $\left\{\bar{X}_{t}\right\}$. The class of processes in (1.3) is quite numerous since different mixing distributions $\Phi$ yield different processes $\left\{\bar{X}_{t}\right\}$ (Proposition 2.2).

The main incentive of our work was answering the question of whether aggregation of the infinite-variance AR(1) series can lead to long memory. To this end, similarly to Zaffaroni (2004), we assume that the mixing distribution is concentrated in the interval $(0,1)$ and has a density $\phi$ such that

$$
\phi(x) \sim \psi(1)(1-x)^{b} \quad \text { as } x \rightarrow 1
$$

for some $\psi(1)>0$ and $b>-1$. In Section 3 we study the long-memory properties of the mixed $\alpha$-stable moving average in (1.3). Clearly, the usual definitions of long memory in 
terms of the covariance/spectrum do not apply in our case. Alternative notions of long memory which are applicable to infinite-variance processes have been proposed in the literature. Among them, we mention the (decay rate of) the codifference (see Samorodnitsky and Taqqu (1994, pp. 103-106)), distributional long memory (see Cox (1984)), and the long-range dependence (sample Allen variance) (LRD(SAV)) property of Heyde and Yang (1997) (see Section 3 for definitions). These three properties are established for the aggregated process $\left\{\bar{X}_{t}\right\}$ in (1.3) under assumption (1.4) in the parameter range

$$
0<b<\alpha-1, \quad 1<\alpha<2 \text {; }
$$

see Theorems 3.1, 3.2, and 3.3, below. In particular, normalized partial sums of $\left\{\bar{X}_{t}\right\}$ in (1.3) tend to an $\alpha$-stable stationary increment process $\{Z(\tau)\}$, which is self-similar with index $H=1-(b / \alpha) \in(1 / \alpha, 1)$ and is written as a stochastic integral

$$
\begin{aligned}
Z(\tau) & :=\int_{\mathbb{R}_{+} \times \mathbb{R}}(f(x, \tau-s)-f(x,-s)) v(\mathrm{~d} x, \mathrm{~d} s), \\
f(x, t) & := \begin{cases}1-\mathrm{e}^{-x t}, & \text { if } x>0 \text { and } t>0, \\
0, & \text { otherwise, }\end{cases}
\end{aligned}
$$

with respect to an independently scattered $\alpha$-stable random measure $v$ on $(0, \infty) \times \mathbb{R}$ with control measure $\psi(1) x^{b-\alpha} \mathrm{d} x \mathrm{~d} s$; see Theorem 3.1 for precise formulations. The value $b=\alpha-1$ seems to separate long memory and short memory in the above aggregation scheme; indeed, in the case $b>\alpha-1$ the aggregated process has the short-range dependence (sample Allen variance) ( $\mathrm{SRD}(\mathrm{SAV})$ ) property and its partial sums tend to an $\alpha$-stable Lévy process with independent increments (see Section 3). Let us note that $\alpha$-stable self-similar processes of the type in (1.5) were discussed in Surgailis et al. (1992), Cioczek-Georges et al. (1995), and Cioczek-Georges and Mandelbrot (1995). Also, note that (1.5) is different from the (more usual) $\alpha$-stable fractional Lévy motion. Since the latter process arises in a similar context by aggregating AR(1) processes with common infinite-variance innovations (see Puplinskaite and Surgailis (2009)), we can conclude that, in the infinite-variance case, the distinctions between dependent and independent aggregation schemes are deeper than in the case of finite variance; see also Remark 2.4, below. On the other hand, there are certain similarities between the two aggregation schemes and long-memory properties of the limiting aggregated processes, including the relation in (3.9), below, between exponents of the mixing density near $a=1$. See Remarks 3.1 and 3.2, below.

The notion of long memory is polysemous, especially for infinite-variance processes, and is not limited to the three characterization properties mentioned above. Samorodnitsky (2004) associated long memory with the rate of growth of maxima and partial maxima of a stationary $\alpha$-stable process. Theorem 4.1 of the above paper says that partial maxima of an $\mathrm{S} \alpha \mathrm{S}$ process generated by a dissipative flow always grow at the rate $n^{1 / \alpha}$. Therefore, the rate of growth of the sequence of partial maxima is incapable of discriminating between long memory and short memory in the aggregate process in (1.3), since this process is a particular case of the class of mixed moving averages generated by dissipative flows. An interesting characterization of long memory by the behavior of ruin probabilities in risk insurance models with $\alpha$-stable claims is given in Mikosch and Samorodnitsky (2000). See Remark 3.3, below.

In what follows, $C$ stands for a constant whose precise value is unimportant and which may

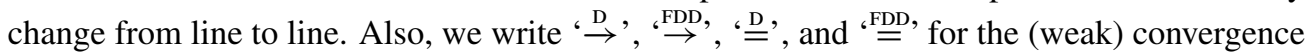
and equality of distributions and finite-dimensional distributions, respectively. The proofs are presented in Appendix A. 


\section{Existence of the limiting aggregated process}

Definition 2.1. Write $\varepsilon \in D(\alpha), 0<\alpha \leq 2$, if

(i) $\alpha=2$ and $\mathrm{E} \varepsilon=0, \sigma^{2}:=\mathrm{E} \varepsilon^{2}<\infty$, or

(ii) $0<\alpha<2$ and there exist some constants $c_{1}, c_{2} \geq 0, c_{1}+c_{2} \neq 0$, such that

$$
\lim _{x \rightarrow \infty} x^{\alpha} \mathrm{P}(\varepsilon>x)=c_{1} \quad \text { and } \quad \lim _{x \rightarrow-\infty}|x|^{\alpha} \mathrm{P}(\varepsilon \leq x)=c_{2} ;
$$

moreover, $\mathrm{E} \varepsilon=0$ whenever $1<\alpha<2$, while, for $\alpha=1$, we assume that the distribution of $\varepsilon$ is symmetric.

Remark 2.1. Condition $\varepsilon \in D(\alpha)$ implies that the RV $\varepsilon$ belongs to the domain of normal attraction of an $\alpha$-stable law; in other words,

$$
n^{-1 / \alpha} \sum_{i=1}^{n} \varepsilon_{i} \stackrel{\mathrm{D}}{\rightarrow} Z,
$$

where $Z$ is an $\alpha$-stable RV; see Feller (1971, pp. 574-581). The symmetry of $\varepsilon$ in the case $\alpha=1$ is not necessary for (2.1) and the subsequent discussion; however, it is imposed to avoid some technical and notational complications. The characteristic function of the RV $Z$ in (2.1) is given by

$$
\mathrm{Ee}^{\mathrm{i} \theta Z}=\mathrm{e}^{-|\theta|^{\alpha} \omega(\theta)}, \quad \theta \in \mathbb{R},
$$

where

$$
\omega(\theta):= \begin{cases}\frac{\Gamma(2-\alpha)}{1-\alpha}\left(\left(c_{1}+c_{2}\right) \cos \left(\frac{\pi \alpha}{2}\right)-\mathrm{i}\left(c_{1}-c_{2}\right) \operatorname{sgn}(\theta) \sin \left(\frac{\pi \alpha}{2}\right)\right), & \alpha \neq 1,2, \\ \left(c_{1}+c_{2}\right) \frac{\pi}{2}, & \alpha=1, \\ \frac{\sigma^{2}}{2}, & \alpha=2 .\end{cases}
$$

The following proposition is easy. See, e.g. Brandt (1986) and Puplinskaite and Surgailis (2009).

Proposition 2.1. Let $\varepsilon \in D(\alpha)$ for some $0<\alpha \leq 2$. Then there exists a unique stationary solution to the $A R(1)$ equation (1.1) given by the series

$$
X_{t}=\sum_{k=0}^{\infty} a^{k} \varepsilon_{t-k} .
$$

The series in (2.3) converges conditionally a.s. and in $L^{p}$ for any $p<\alpha$ and almost every $a \in(-1,1)$. Moreover, if

$$
\mathrm{E}\left[\frac{1}{1-|a|^{p}}\right]<\infty
$$

for some $0<p<\alpha$, then the series in (2.3) converges unconditionally in $L^{p}$. 
Let the $\left\{X_{i t}\right\}, i=1,2, \ldots$, be independent copies of (2.3). We are interested in the existence and properties of the limiting aggregated process $\left\{\bar{X}_{t}\right\}$ as defined by (1.2).

Introduce an independently scattered $\alpha$-stable random measure $M=\left\{M_{s}(\mathrm{~d} a), s \in \mathbb{Z}, a \in\right.$ $(-1,1)\}$ on $\mathbb{Z} \times(-1,1)$ with the characteristic functional

$$
\operatorname{E} \exp \left\{\mathrm{i} \sum_{s \in \mathbb{Z}} \theta_{s} M_{S}\left(A_{S}\right)\right\}=\exp \left\{-\sum_{s \in \mathbb{Z}}\left|\theta_{s}\right|^{\alpha} \omega\left(\theta_{s}\right) \Phi\left(A_{s}\right)\right\},
$$

where $\theta_{s} \in \mathbb{R}$ and $A_{s} \subset(-1,1)$ are arbitrary Borel sets.

Theorem 2.1. Let $\varepsilon \in D(\alpha)$ for some $0<\alpha \leq 2$, and let condition (2.4) be satisfied. Then the limiting aggregated process $\left\{\bar{X}_{t}\right\}$ in (1.2) exists. It is stationary, ergodic, has $\alpha$-stable finite-dimensional distributions, and a stochastic integral representation as in (1.3), where $M$ is an $\alpha$-stable random measure as defined in (2.5).

Remark 2.2. If the distribution $\Phi$ is concentrated at a finite number of points $a_{1}, \ldots, a_{k} \in$ $(-1,1)$ and $\phi_{i}:=\mathrm{P}\left(a=a_{i}\right)>0$, the process in (1.3) can be written as a sum of independent $\alpha$-stable $\operatorname{AR}(1)$ processes:

$$
\bar{X}_{t}=\sum_{i=1}^{k} Y_{i t}, \quad Y_{i t}:=\sum_{s \leq t} a_{i}^{t-s} \zeta_{i s}
$$

where $\left\{\zeta_{i s}:=M_{s}\left(\left\{a_{i}\right\}\right), s \in \mathbb{Z}\right\}$ is an i.i.d. sequence of $\alpha$-stable RVs with $\mathrm{E} \mathrm{e}^{\mathrm{i} \zeta_{i s} \theta}=\mathrm{e}^{-|\theta|{ }^{\alpha} \omega(\theta) \phi_{i}}$. For a general mixing distribution $\Phi$, the process in (1.3) can be approximated by finite sums of $\mathrm{AR}(1)$ processes as in (2.6). The process in (1.3) is well defined (see Surgailis et al. (1993)) if and only if

$$
\sum_{s \in \mathbb{Z}} \mathrm{E}\left|a^{t-s}\right|^{\alpha} \mathbf{1}(s \leq t)=\sum_{k=0}^{\infty} \mathrm{E}|a|^{\alpha k}=\mathrm{E}\left[\frac{1}{1-|a|^{\alpha}}\right]<\infty,
$$

which agrees with (2.4). The characteristic function of (1.3) is given by

$$
\operatorname{Eexp}\left\{\mathrm{i} \sum_{t=1}^{m} \theta_{t} \bar{X}_{t}\right\}=\exp \left\{-\sum_{s \in \mathbb{Z}} \mathrm{E}\left[\left|\sum_{t=1}^{m} \theta_{t} a^{t-s} \mathbf{1}(s \leq t)\right|^{\alpha} \omega\left(\sum_{t=1}^{m} \theta_{t} a^{t-s} \mathbf{1}(s \leq t)\right)\right]\right\} .
$$

Remark 2.3. For $\alpha=2$, the limit process in (1.3) is Gaussian and its covariance function is given by

$$
\operatorname{cov}\left(\bar{X}_{0}, \bar{X}_{t}\right)=\sigma^{2} \sum_{s \leq 0} \int_{(-1,1)} a^{t-s} a^{-s} \Phi(\mathrm{d} a)=\sigma^{2} \mathrm{E} \frac{a^{t}}{1-a^{2}}=\operatorname{cov}\left(X_{0}, X_{t}\right)
$$

and coincides with the covariance of the original series in (2.3). For $\alpha=2$, the statement of Theorem 2.1 is well known; see Oppenheim and Viano (2004) and Zaffaroni (2004).

It is clear from (2.7) that the distribution (i.e. finite-dimensional distribution) of $\left\{\bar{X}_{t}\right\}$ is uniquely determined by the distributions of the RVs $a$ and $Z$ in (2.1). It is also clear that the distribution of $\left\{\bar{X}_{t}\right\}$ (particularly, the marginal $\alpha$-stable distribution of $\bar{X}_{0}$ ) uniquely determines the parameter $\alpha$. Part (i) of Proposition 2.2, below, shows that the class of mixed stable moving averages in (1.3) is nonparametric and very large, since different mixing distributions lead to different processes. Part (ii) says that this class is different from (usual) $\alpha$-stable moving averages, except for a trivial mixing distribution $\Phi$. 
Proposition 2.2. Let $0<\alpha<2$.

(i) The distribution of $\left\{\bar{X}_{t}\right\}$ in (1.3) uniquely determines the distribution $\Phi$.

(ii) Let $\left\{\bar{X}_{t}\right\} \stackrel{\mathrm{FDD}}{=}\left\{Y_{t}\right\}, Y_{t}:=\sum_{j=0}^{\infty} c_{j} \zeta_{t-j}$, where $\left\{\zeta_{t}\right\}$ is an i.i.d. sequence having the same distribution as the $\alpha$-stable $R V$ in (2.1), and let $c_{j}, j \geq 0$, be real coefficients with $\sum_{j=0}^{\infty}\left|c_{j}\right|^{\alpha}<\infty$. Then there exist $a_{0} \in(-1,1)$ and $\epsilon \in\{-1,1\}$ such that $c_{j}=\epsilon a_{0}^{j}$ and $\Phi=\delta_{a_{0}}$.

Let us note that condition (2.4) is crucial for the existence of the nontrivial limit of aggregated $\mathrm{AR}(1)$ processes. Note also that condition (2.4) does not depend on $p>0$ since

$$
\sup _{0 \leq a<1} \frac{1-a^{q}}{1-a^{p}}<\infty
$$

for any $p, q>0$. Below we show that if condition (2.4) is violated and the mixing density has a power-law behavior at $a=1$ with negative exponent $b \in(-1,0)$, the limit aggregated process is a random $\alpha(1+b)$-stable constant whose stability index $\alpha(1+b)<\alpha$. For notational simplicity, we assume that the noise belongs to the domain of attraction of a symmetric $\alpha$-stable law.

Proposition 2.3. Assume that $\varepsilon \in D(\alpha), 0<\alpha \leq 2$, and that $\omega(\theta) \equiv 1$ in (A.1). Moreover, assume that the mixing density has the form

$$
\phi(a)=\psi(a)(1-a)^{b}, \quad a \in(0,1),
$$

where $b \in(-1,0)$ and $\psi$ is an integrable function on $(0,1)$ having a limit

$$
\psi(1):=\lim _{a \rightarrow 1} \psi(a)>0 .
$$

Then

$$
N^{-1 / \alpha(1+b)} \sum_{i=1}^{N} X_{i t} \stackrel{\text { FDD }}{\rightarrow} \tilde{Z},
$$

where the limit process $\tilde{Z}$ does not depend on $t$ and is an $\alpha(1+b)$-stable $R V$ with characteristic function $\mathrm{E}^{\mathrm{i} \theta \tilde{Z}}=\mathrm{e}^{-K|\theta|^{\alpha(1+b)}}$, where $K$ is given in (A.9).

Note that, for the mixing density in (2.9) with $b>0$, Theorem 2.1 applies and, therefore, $b=0$ is a critical point resulting in completely different limits of the aggregated process in the cases $b>0$ and $b<0$. The fact that the limit is degenerate in the latter case can be explained as follows. It is clear that, with $b$ decreasing, the dependence increases in the random-coefficient $\operatorname{AR}(1)$ process $\left\{X_{t}\right\}$, as well as in the limiting aggregated process $\left\{\bar{X}_{t}\right\}$. In Section 3 we show that the dependence in $\left\{\bar{X}_{t}\right\}$ decays hyperbolically with the lag, with an exponent which depends on $b$ and $\alpha$ and which tends to 0 as $b \downarrow 0$. Therefore, for negative $b<0$, the dependence in the aggregated process becomes extremely strong so that the limit process is degenerate and completely dependent.

Remark 2.4. Let $M$ be the $\alpha$-stable random measure in (2.5), and let $\left\{\zeta_{s}:=M_{s}(-1,1), s \in \mathbb{Z}\right\}$ be the corresponding i.i.d. sequence of $\alpha$-stable RVs. Let $\left\{\bar{X}_{t}\right\}$ be the aggregated mixed $\alpha$-stable moving average in (1.3), and let $1<\alpha \leq 2$. Then

$$
\mathrm{E}\left[\bar{X}_{t} \mid \zeta_{s}, s \in \mathbb{Z}\right]=\sum_{s \leq t} \mathrm{E}\left[a^{t-s}\right] \zeta_{s}, \quad t \in \mathbb{Z} .
$$


Relation (2.10) follows from a general 'interpolation formula' for independently scattered random measures (see Surgailis (1979, Proposition 1.3)). For the reader's convenience, we present this formula for the $\alpha$-stable measure $M$ in Proposition 2.4, below. Recall from Puplinskaite and Surgailis (2009) that the right-hand side of (2.10) represents the limiting aggregated process in the $\mathrm{AR}(1)$ aggregation scheme with common $\alpha$-stable innovations $\varepsilon_{s}=\zeta_{s}$, $s \in \mathbb{Z}$. Thus, (2.10) establishes a link between the aggregated processes in the two aggregation schemes. It also suggests that the latter aggregation scheme leads to a simpler aggregated process when compared to process (1.3) in the present paper. In particular, the moving average on the right-hand side of (2.10) may be invertible (which occurs, e.g. in the case of FARIMA $(0, d, 0)$ coefficients $\mathrm{E}\left[a^{t-s}\right]$ mentioned in the introduction), while, for the mixed moving average in (1.3), the usual definition of invertibility does not apply and the possibility of 'recovering' $M_{t}(A)$ from $X_{s}, s \leq t$, seems unlikely. On the other hand, in the finite-variance case, $\alpha=2$, the aggregated process $\left\{\bar{X}_{t}\right\}$ is Gaussian with covariance given in (2.8); hence, it is also invertible under known conditions on the spectral density. (A particular form of the mixing density $\phi$ leading to the $\operatorname{FARIMA}(0, d, 0)$ Gaussian process $\left\{\bar{X}_{t}\right\}$ was found in Celov et al. (2007).) The above discussion complies with the remark in the introduction that the distinctions between dependent and independent aggregation schemes in the infinite-variance case are deeper than in the finite-variance case.

Let $L_{\alpha}(\mathbb{Z} \times(-1,1))$ denote the class of all measurable functions $h: \mathbb{Z} \times(-1,1) \rightarrow \mathbb{R}$ with

$$
\sum_{t \in \mathbb{Z}} \mathrm{E}|h(s, a)|^{\alpha}<\infty, \quad 1<\alpha \leq 2 .
$$

The stochastic integral

$$
M(h):=\sum_{s \in \mathbb{Z}} \int_{(-1,1)} h(s, a) M_{s}(\mathrm{~d} a)
$$

is well defined for any $h \in L_{\alpha}(\mathbb{Z} \times(-1,1))$; see Samorodnitsky and Taqqu (1994, pp. 111-167).

Proposition 2.4. Let $M$ and $\left\{\zeta_{s}, s \in \mathbb{Z}\right\}$ be the same as in Remark 2.4, and let $1<\alpha \leq 2$. Then, for any $h \in L_{\alpha}(\mathbb{Z} \times(-1,1))$,

$$
\mathrm{E}\left[M(h) \mid \zeta_{s}, s \in \mathbb{Z}\right]=\sum_{s \in \mathbb{Z}} \bar{h}(s) \zeta_{s} ; \quad \bar{h}(s):=\mathrm{E} h(s, a) .
$$

\section{Long-memory properties of the aggregated process}

Definition 3.1. A strictly stationary time series $\left\{Y_{t}\right\}$ is said to have distributional long memory or distributional short memory if there exist some constants $A_{n} \rightarrow \infty(n \rightarrow \infty)$ and $B_{n}$, and a stochastic process $\{J(t), t \geq 0\} \not \equiv 0$ with dependent increments or, respectively, independent increments such that

$$
A_{n}^{-1} \sum_{s=1}^{[n t]}\left(Y_{s}-B_{n}\right) \stackrel{\text { FDD }}{\rightarrow} J(t) .
$$

Definition 3.1 is due to Cox (1984). Lamperti (1962) showed that, under mild additional assumptions, the normalizing constants $A_{n}$ in (3.1) grow as $n^{H}$ with some $H>0$; more precisely, $A_{n}=L(n) n^{H}$, where $L(n)$ is a slowly varying function at $\infty$, and the limit process $\{J(t), t \geq 0\}$ is self-similar with index $H$. 
Recall the definition of the process $\{Z(\tau), \tau \in \mathbb{R}\}$ in (1.5). This process is well defined for any $0<b<\alpha-1$ and $\alpha \in(1,2)$, and its characteristic functional is given by

$$
\begin{aligned}
\operatorname{Eexp}\left\{\mathrm{i} \sum_{i=1}^{m} \theta_{i} Z\left(\tau_{i}\right)\right\} & \\
=\exp \left\{-\psi(1) \int_{\mathbb{R}} \int_{\mathbb{R}_{+}} \mid\right. & \left.\sum_{i=1}^{m} \theta_{i}\left(f\left(x, \tau_{i}-s\right)-f(x,-s)\right)\right|^{\alpha} \\
& \left.\times \omega\left(\sum_{i=1}^{m} \theta_{i}\left(f\left(x, \tau_{i}-s\right)-f(x,-s)\right)\right) x^{b-\alpha} \mathrm{d} s \mathrm{~d} x\right\},
\end{aligned}
$$

where $\tau_{i}, \theta_{i} \in \mathbb{R}, i=1, \ldots, m, m=1,2, \ldots$ The process $\{Z(\tau)\}$ is self-similar with index

$$
H=1-\frac{b}{\alpha} \in\left(\frac{1}{\alpha}, 1\right),
$$

which follows from (3.2) by the change of variables $s \rightarrow \lambda s, x \rightarrow x / \lambda(\lambda>0)$, and has $\alpha$-stable finite-dimensional distributions and stationary increments. From these facts and Kolmogorov's moment criterion, it follows that $\{Z(\tau)\}$ has a sample continuous version. See also Surgailis et al. (1992, Corollary 4).

Theorem 3.1. Let $\left\{\bar{X}_{t}\right\}$ be the aggregated process in (2.1) with mixing density as in (2.9), where $b>0$ and $\psi$ is integrable on $(0,1)$ and has a limit $\lim _{a \rightarrow 1-} \psi(a)=: \psi(1)>0$.

(i) Let $1<\alpha<2$ and $0<b<\alpha-1$. Let $H=1-b / \alpha$, as in (3.3). Then

$$
\frac{1}{n^{H}} \sum_{t=1}^{[n \tau]} \bar{X}_{t} \stackrel{\text { FDD }}{\rightarrow} Z(\tau)
$$

where the limit process is given in (1.5).

(ii) Let $0<\alpha<2$ and $b>\max (\alpha-1,0)$. Then

$$
\frac{1}{n^{1 / \alpha}} \sum_{t=1}^{[n \tau]} \bar{X}_{t} \stackrel{\mathrm{FDD}}{\rightarrow} L(\tau)
$$

where $\{L(\tau), \tau \geq 0\}$ is an $\alpha$-stable homogeneous Lévy process with characteristic function

$$
\mathrm{Ee}^{\mathrm{i} \theta L(\tau)}=\mathrm{e}^{-K|\theta|^{\alpha} \omega(\theta) \tau}, \quad K:=\int_{0}^{1}(1-x)^{-\alpha} \phi(x) \mathrm{d} x .
$$

Since the process $\{Z(\tau)\}$ in (3.4) has dependent increments while the Lévy process $\{Z(\tau)\}$ in (3.5) has independent increments, from Theorem 3.1 we conclude that the aggregated process $\left\{\bar{X}_{t}\right\}$ with mixing density as in (2.9) has distributional long memory for $0<b<\alpha-1,1<$ $\alpha<2$, and distributional short memory for $b>\max (\alpha-1,0)$.

Next, we turn to the study of the LRD(SAV) property, as defined in Heyde and Yang (1997).

Definition 3.2. (i) A strictly stationary zero-mean process $\left\{Y_{t}, t \in \mathbb{Z}\right\}$ is called $\mathrm{LRD}(\mathrm{SAV})$ if

$$
\frac{\left(\sum_{t=1}^{n} Y_{t}\right)^{2}}{\sum_{t=1}^{n} Y_{t}^{2}}
$$

tends to $\infty$ in probability as $n \rightarrow \infty$. 
(ii) A strictly stationary zero-mean process $\left\{Y_{t}, t \in \mathbb{Z}\right\}$ is called $\operatorname{SRD}(\mathrm{SAV})$ if the ratio in (3.6) is bounded in probability.

Theorem 3.2. Let $\left\{\bar{X}_{t}\right\}$ satisfy the conditions of Theorem 3.1.

(i) Let $1<\alpha<2$ and $0<b<\alpha-1$. Then $\left\{\bar{X}_{t}\right\}$ is $\operatorname{LRD}(\operatorname{SAV})$.

(ii) Let $1<\alpha<2$ and $b>\alpha-1$. Then $\left\{\bar{X}_{t}\right\}$ is $\operatorname{SRD}(\operatorname{SAV})$.

For a strictly stationary process $\left\{Y_{t}\right\}$, let

$$
U_{Y}\left(\theta_{1}, \theta_{2} ; t\right):=\operatorname{cov}\left(\mathrm{e}^{\mathrm{i} \theta_{1} Y_{t}}, \mathrm{e}^{\mathrm{i} \theta_{0} Y_{0}}\right)=\mathrm{Ee}^{\mathrm{i}\left(\theta_{1} Y_{t}-\theta_{2} Y_{0}\right)}-\mathrm{Ee}^{\mathrm{i} \theta_{1} Y_{t}} \mathrm{Ee}^{-\mathrm{i} \theta_{2} Y_{0}} .
$$

The long memory of $\left\{Y_{t}\right\}$ can be characterized by the decay rate of $U_{Y}\left(\theta_{1}, \theta_{2} ; t\right)$ as $t \rightarrow \infty$. Note that the existence of $U_{Y}\left(\theta_{1}, \theta_{2} ; t\right)$ does not require any moments. For stationary stable or heavy-tailed moving averages and some other processes with long memory, the asymptotics of $U_{Y}\left(\theta_{1}, \theta_{2} ; t\right)$ were investigated in Astrauskas (1983), Astrauskas et al. (1991), and Koul and Surgailis (2001). A related quantity,

$$
\operatorname{cod}\left(Y_{0}, Y_{t}\right):=\log \mathrm{E} \mathrm{e}^{\mathrm{i}\left(Y_{t}-Y_{0}\right)}-\log \mathrm{E}^{\mathrm{i} Y_{t}}-\log \mathrm{Ee}^{-\mathrm{i} Y_{0}},
$$

called the codifference of the RVs $Y_{0}$ and $Y_{t}$, can also be used to characterize the long memory of $\left\{Y_{t}\right\}$ and its intensity (see Samorodnitsky and Taqqu (1994), pp. 384-387). In particular, if $\left\{Y_{t}\right\}$ is a stationary Gaussian process, with zero mean, unit variance, and covariance $r(t) \rightarrow$ $0(t \rightarrow \infty)$, then $\operatorname{cod}\left(Y_{0}, Y_{t}\right)=\frac{1}{2} \operatorname{cov}\left(Y_{0}, Y_{t}\right)=\frac{1}{2} r(t)$, while

$$
U_{Y}\left(\theta_{1}, \theta_{2} ; t\right)=\mathrm{e}^{-\left(\theta_{1}^{2}+\theta_{2}^{2}\right) / 2}\left(\mathrm{e}^{\theta_{1} \theta_{2} r(t)}-1\right) \sim \theta_{1} \theta_{2} \mathrm{e}^{-\left(\theta_{1}^{2}+\theta_{2}^{2}\right) / 2} r(t) \quad \text { as } t \rightarrow \infty
$$

decays as $r(t)$.

Theorem 3.3, below, gives the decay rate of the codifference of the mixed stable moving average in (1.3) and the mixing density in (3.7), below. A similar result can be proved for the covariance $U_{\bar{X}}\left(\theta_{1}, \theta_{2} ; t\right)$. Write $\bar{z}$ for the conjugate of a complex number $z \in \mathbb{C}$.

Theorem 3.3. Let $\left\{\bar{X}_{t}\right\}$ be the aggregated process in (1.3), with characteristic functional as in (2.7), $0<\alpha<2$, and mixing density

$$
\phi(a)=\psi(a)\left\{\begin{array}{ll}
(1-a)^{b_{1}}, & 0<a<1, \\
(1+a)^{b_{2},}, & -1<a<0,
\end{array} \quad a \in(-1,1)\right.
$$

where $1>b_{1}, b_{2}>0$ are parameters and $\psi$ is continuous at \pm 1 with $\lim _{a \rightarrow \pm 1} \psi(a)=$ : $\psi( \pm 1) \geq 0$. Then, as $t \rightarrow \infty$,

$$
\operatorname{cod}\left(\bar{X}_{0}, \bar{X}_{t}\right)=\left(C_{1}+o(1)\right) t^{-b_{1}}+\left(C_{2}(t)+o(1)\right) t^{-b_{2}},
$$

where

$$
\begin{gathered}
C_{1}:=\psi(1) \alpha^{-1} \int_{0}^{\infty}\left[\omega(1) \mathrm{e}^{-y \alpha}+\overline{\omega(1)}\left(1-\left(1-\mathrm{e}^{-y}\right)^{\alpha}\right)\right] y^{b_{1}-1} \mathrm{~d} y, \\
C_{2}(t):=\psi(-1) \alpha^{-1} \operatorname{Re}(\omega(1)) \int_{0}^{\infty}\left[\mathrm{e}^{-y \alpha}+1-\left(1-(-1)^{t} \mathrm{e}^{-y}\right)^{\alpha}\right] y^{b_{2}-1} \mathrm{~d} y .
\end{gathered}
$$


Remark 3.1. For $1<\alpha \leq 2$ and $0<b<\alpha-1$, introduce the parameter

$$
d:=\frac{\alpha-1-b}{\alpha},
$$

or $b=\alpha-1-\alpha d$. Note that $b=0$ if and only if $d=1-1 / \alpha$, and $b=\alpha-1$ if and only if $d=0$. Recall from Samorodnitsky and Taqqu (1994, Theorem 7.13.4) that, for the $\operatorname{FARIMA}(0, d, 0)$ process $\left\{Y_{t}\right\}$ with $\alpha$-stable innovations, $0<d<1-1 / \alpha$, and $1<\alpha \leq 2$,

$$
\operatorname{cod}\left(Y_{0}, Y_{t}\right) \sim(\text { constant }) t^{1+\alpha d-\alpha} \text { as } t \rightarrow \infty .
$$

Therefore, Theorem 3.3 implies that the codifference of the aggregated process $\left\{\bar{X}_{t}\right\}$ in (1.3) with the mixing density in (2.9) and $0<b<\alpha-1$ decays similarly as the codifference of an $\alpha$-stable FARIMA $(0, d, 0)$ process with parameter $d$ given in (3.9). From Theorem 3.1 we see that the above similarity between $\left\{\bar{X}_{t}\right\}$ and FARIMA $(0, d, 0)$ with parameter $d$ in (3.9) also extends to the normalization exponent $H$ of partial sums of both processes: for the former process, $H=1-b / \alpha$ and, for the latter process, $H=d+1 / \alpha$. Clearly, $1-b / \alpha=d+1 / \alpha$ is equivalent to (3.9). In other words, if $b$ and $d$ are related as in (3.9) then partial sums of $\left\{\bar{X}_{t}\right\}$ and partial sums of the FARIMA $(0, d, 0)$ process converge under the same normalization and the limits are self-similar processes with the same parameter $H$.

Remark 3.2. Recall that a second-order stationary process is said to have covariance long memory if the sum of the absolute values of covariances diverges. In the case of an infinitevariance process, the divergence of the absolute values of codifferences also indicates the presence of long memory. From Theorems 3.1-3.3 we see that the codifference of $\left\{\bar{X}_{t}\right\}$ is nonsummable for any $0<b<1$, irrespective of the value of $\alpha$, while at the same time this process may have the $\operatorname{SRD}(\mathrm{SAV})$ property and distributional short memory, provided $\alpha-1<$ $b<1$ and $1<\alpha<2$. These results might look strange and a peculiarity of the process in (1.3) at first glance; however, similar facts also hold for moving averages $Y_{t}=\sum_{j=0}^{\infty} c_{j} \varepsilon_{t-j}$ in i.i.d. innovations $\varepsilon_{t} \in D(\alpha)$ with regularly decaying coefficients $c_{j} \sim j^{d-1}$. Indeed, for such $\left\{Y_{t}\right\}$, the codifference decays as in (3.10) for any $0<\alpha<2$ and $d<1-1 / \alpha$, so that $\sum_{j=0}^{\infty}\left|\operatorname{cod}\left(Y_{0}, Y_{j}\right)\right|=\infty$ and $\sum_{j=0}^{\infty}\left|c_{j}\right|<\infty$ hold for $1-2 / \alpha<d<0$. Since $\left\{Y_{t}\right\}$ has distributional short memory for $d<0$ and $\sum_{j=0}^{\infty} c_{j} \neq 0$ (see, e.g. Astrauskas (1983)), we have exactly the same situation as in the case of $\left\{X_{t}\right\}$, with parameters $d$ and $b$ related as in (3.9).

Remark 3.3. Mikosch and Samorodnitsky (2000) discussed the asymptotic behavior of the ruin probability

$$
\psi(u):=\mathrm{P}\left(\sup _{n \geq 0}\left(X_{1}+\cdots+X_{n}-n \mu\right)>u\right)
$$

as $u \rightarrow \infty$, where 'claims' $\left\{X_{t}\right\}$ form a stationary $\alpha$-stable process, $1<\alpha<2$, and $\mu>\mathrm{E} X_{1}$ is a given constant. They associated the 'classical' decay rate $\psi(u)=O\left(u^{-(\alpha-1)}\right)$ with shortrange dependence and the decay rate $\psi(u)=O\left(u^{-v}\right)$ with exponent $v<\alpha-1$ with long-range dependence of the claim sequence $\left\{X_{t}\right\}$. In the case when the $X_{t} \mathrm{~s}$ are stationary increments of a linear $\alpha$-stable fractional motion with self-similarity parameter $H \in(1 / \alpha, 1)$, Mikosch and Samorodnitsky (2000, Proposition 4.4) obtained a decay rate $\psi(u) \sim($ constant $) u^{-\alpha(1-H)}$ of the ruin probability. Let us note that increments of an $\alpha$-stable fractional motion satisfy the distributional long-memory property and also exhibit the decay of codifference as in (3.10), with $d$ and $H$ related as in Remark 3.1 (see Samorodnitsky and Taqqu (1994, pp. 380-387)). Therefore, the above characterization of long memory via ruin probabilities seems to agree with 
other characterizations of long memory discussed in this paper, at least for $\alpha$-stable moving averages. An interesting open problem is to extend the asymptotics of the ruin probability from Mikosch and Samorodnitsky (2000, Proposition 4.4) to the aggregate process $\left\{\bar{X}_{t}\right\}$ in Theorem 3.1.

\section{Appendix A. Proofs}

Proof of Theorem 2.1. The characteristic function of the RV $\varepsilon \in D(\alpha)$ has the following representation in a neighborhood of the origin (see, e.g. Ibragimov and Linnik (1971, Theorem 2.6.5)): there exists an $\epsilon>0$ such that

$$
\mathrm{Ee}^{\mathrm{i} \theta \varepsilon}=\mathrm{e}^{-|\theta|^{\alpha} \omega(\theta) h(\theta)}, \quad|\theta|<\epsilon,
$$

where $h$ is a positive function tending to 1 as $\theta \rightarrow 0$. Denote

$$
\vartheta(s, a):=\sum_{t=1}^{m} \theta_{t} a^{t-s} \mathbf{1}(s \leq t) .
$$

Then $N^{-1 / \alpha} \sum_{t=1}^{m} \theta_{t} X_{t}=N^{-1 / \alpha} \sum_{s \in \mathbb{Z}} \vartheta(s, a) \varepsilon_{s}$. Since $m$ and $\theta_{t}, t=1, \ldots, m$, are fixed and $a$ is bounded, it is clear that $|\vartheta(s, a)| \leq C$ for a constant $C$ independent of $a$ and $s$, and, therefore, $\left|N^{-1 / \alpha} \vartheta(s, a)\right|<\epsilon$ for all $N>N_{0}$ large enough. Therefore, using (A.1), we can write

$$
\begin{aligned}
\mathrm{E} \exp & \left\{\mathrm{i} N^{-1 / \alpha} \sum_{i=1}^{N} \sum_{t=1}^{m} \theta_{t} X_{i t}\right\} \\
& =\left(\operatorname{E} \exp \left\{\mathrm{i} N^{-1 / \alpha} \sum_{t=1}^{m} \theta_{t} X_{t}\right\}\right)^{N} \\
& =\left(\operatorname{E} \exp \left\{-N^{-1} \sum_{s \in \mathbb{Z}}|\vartheta(s, a)|^{\alpha} h\left(N^{-1 / \alpha} \vartheta(s, a)\right) \omega(\vartheta(s, a))\right\}\right)^{N} .
\end{aligned}
$$

Clearly, for any $a \in(-1,1)$,

$$
\sum_{s \in \mathbb{Z}}|\vartheta(s, a)|^{\alpha} h\left(N^{-1 / \alpha} \vartheta(s, a)\right) \omega(\vartheta(s, a)) \rightarrow \sum_{s \in \mathbb{Z}}|\vartheta(s, a)|^{\alpha} \omega(\vartheta(s, a))
$$

as $N \rightarrow \infty$, and

$$
\left.\left|\sum_{s \in \mathbb{Z}}\right| \vartheta(s, a)\right|^{\alpha} h\left(N^{-1 / \alpha} \vartheta(s, a)\right) \omega(\vartheta(s, a)) \mid \leq \frac{C}{1-|a|^{\alpha}}
$$

for a constant $C<\infty$ independent of $a$. Define

$$
\Theta_{N}:=N \mathrm{E}\left[\exp \left\{-N^{-1} \sum_{s \in \mathbb{Z}}|\vartheta(s, a)|^{\alpha} h\left(N^{-1 / \alpha} \vartheta(s, a)\right) \omega(\vartheta(s, a))\right\}-1\right] .
$$

Using (A.3), (A.4), condition (2.4), the fact that $0 \leq h(\theta) \leq C$, the inequality $\left|\mathrm{e}^{z}-1\right| \leq|z|$ $(z \in \mathbb{C}, \operatorname{Re} z \leq 0)$, and the dominated convergence theorem, we obtain

$$
\lim _{N \rightarrow \infty} \Theta_{N}=-\sum_{s \in \mathbb{Z}} \mathrm{E}\left[|\vartheta(s, a)|^{\alpha} \omega(\vartheta(s, a))\right] .
$$


Therefore,

$$
\begin{aligned}
\lim _{N \rightarrow \infty} \operatorname{Eexp}\left\{\mathrm{i} N^{-1 / \alpha} \sum_{i=1}^{N} \sum_{t=1}^{m} \theta_{t} X_{i t}\right\} & =\lim _{N \rightarrow \infty}\left(1+\frac{\Theta_{N}}{N}\right)^{N} \\
& =\exp \left\{-\sum_{s \in \mathbb{Z}} \mathrm{E}\left[|\vartheta(s, a)|^{\alpha} \omega(\vartheta(s, a))\right]\right\},
\end{aligned}
$$

which coincides with (2.7). The properties of $\left\{\bar{X}_{t}\right\}$ mentioned in the statement of the theorem follow from Surgailis et al. (1993). This completes the proof.

Proof of Proposition 2.2. (i) By separately considering the real and imaginary parts of the logarithm of the characteristic function in (2.7), we see that it suffices to prove the proposition for the symmetric case $\omega \equiv 1$ only.

Let $L_{\alpha}(\mathbb{Z})$ be the space of all real sequences $g=\left(g_{t}, t \in \mathbb{Z}\right)$ with $\|g\|_{\alpha}^{\alpha}:=\sum_{t \in \mathbb{Z}}\left|g_{t}\right|^{\alpha}<\infty$. Let $\mathcal{B}\left(L_{\alpha}(\mathbb{Z})\right)$ be the $\sigma$-algebra of Borel sets of $L_{\alpha}(\mathbb{Z})$. A Borel set $A \subset L_{\alpha}(\mathbb{Z})$ is said to be symmetric if $-A=A$ and shift invariant if $U_{t} A=A$ for every $t \in \mathbb{Z}$, where $U_{s}, s \in \mathbb{Z}$, is the group of shift operators on $L_{\alpha}(\mathbb{Z}),\left(U_{s} g\right)_{t}:=g_{t-s}$. Let $\mathcal{B}_{\text {inv }}\left(L_{\alpha}(\mathbb{Z})\right)$ denote the class of all open symmetric and shift-invariant sets $A \subset L_{\alpha}(\mathbb{Z})$.

According to Surgailis et al. (1993, Theorem 2 and Lemma 1), the characteristic function in (2.7) uniquely determines the measure

$$
\mu(A):=\int_{L_{\alpha}(\mathbb{Z})} \mathbf{1}\left(\frac{g}{\|g\|_{\alpha}} \in A\right)\|g\|_{\alpha}^{\alpha} \lambda(\mathrm{d} g),
$$

on open symmetric and shift-invariant sets $A \in \mathcal{B}_{\text {inv }}\left(L_{\alpha}(\mathbb{Z})\right)$ and vice versa; here

$$
\lambda(A):=\mathrm{P}\left(\left(a^{-t} \mathbf{1}(t \leq 0), t \in \mathbb{Z}\right) \in A\right), \quad A \in \mathcal{B}\left(L_{\alpha}(\mathbb{Z})\right),
$$

is a probability measure concentrated on the set $\left\{g=\left(g_{t}, t \in \mathbb{Z}\right) \in L_{\alpha}(\mathbb{Z}): g_{t}=a^{-t} \mathbf{1}(t \leq 0)\right.$, there exists $a \in(-1,1)\}$ of geometric progressions.

Let $V \subset(-1,1)$ be an open set, and let

$$
\begin{aligned}
A(V) & :=\bigcup_{s \in \mathbb{Z}} \bigcup_{\delta= \pm 1} A_{s, \delta}(V), \\
A_{s, \delta}(V) & :=\left\{f=\left(f_{t}, t \in \mathbb{Z}\right) \in L_{\alpha}(\mathbb{Z}): f_{t}=\delta\left(1-|v|^{\alpha}\right)^{1 / \alpha} v^{s-t} \mathbf{1}(t \leq s),\right.
\end{aligned}
$$

there exists $v \in V\}$.

Note that, $A_{s, \delta}(V)$ are disjoint sets for distinct pairs $(s, \delta)$, the set $A(V)$ is open, symmetric and shift invariant, and $\mu\left(A_{s, \delta}(V)\right)=0$ unless $(s, \delta)=(0,1)$. Moreover,

$$
\mu(A(V))=\mu\left(A_{(0,1)}(V)\right)=\mathrm{E}\left[\frac{\mathbf{1}(a \in V)}{1-|a|^{\alpha}}\right]=\int_{V} \frac{\Phi(\mathrm{d} a)}{1-|a|^{\alpha}}=: G(V)
$$

according to the definitions in (A.5)-(A.6). Therefore, the characteristic function in (2.7) uniquely determines the measure $G$ on the interval $(-1,1)$. Since $\Phi(V)=\int_{V}\left(1-|a|^{\alpha}\right) G(\mathrm{~d} a)$, part (i) of the proposition follows. let

(ii) As in (i), it suffices to discuss the case $\omega \equiv 1$. Let $\mu=\mu_{\bar{X}}$ be as defined in (A.5), and

$$
\mu_{Y}(A):=\|c\|_{\alpha}^{\alpha} \mathbf{1}\left(\frac{c}{\|c\|_{\alpha}} \in A\right), \quad c:=\left(c_{-t} \mathbf{1}(t \leq 0), t \in \mathbb{Z}\right) \in L_{\alpha}(\mathbb{Z}),
$$


be the measure on the unit sphere of $L_{\alpha}(\mathbb{Z})$, corresponding to the moving average $\left\{Y_{t}\right\}$. By definition, $\mu_{Y}$ is concentrated on a single element $c /\|c\|_{\alpha} \in L_{\alpha}(\mathbb{Z})$.

As mentioned above in the proof of (i), $\left\{\bar{X}_{t}\right\} \stackrel{\text { FDD }}{=}\left\{Y_{t}\right\}$ implies that

$$
\mu_{Y}(A)=\mu_{\bar{X}}(A), \quad A \in \mathcal{B}_{\text {inv }}\left(L_{\alpha}(\mathbb{Z})\right) .
$$

Consider the set $A=A(-1,1)$, as defined in (A.7), consisting of all signed translations of normalized geometric progressions. Clearly, $c /\|c\|_{\alpha} \in A(-1,1)$ if and only if $c_{j}=\epsilon a_{0}^{j}$, $j \geq 0$, for some $a_{0} \in(-1,1)$ and $\epsilon \in\{-1,1\}$. It also easily follows from (A.8) that $\Phi=\delta_{a_{0}}$. This completes the proof.

Proof of Proposition 2.3. Let

$$
\Theta_{N}:=N \mathrm{E}\left[\exp \left\{-N^{-1 /(1+b)} \sum_{s \in \mathbb{Z}}|\vartheta(s, a)|^{\alpha} h\left(N^{-1 / \alpha(1+b)} \vartheta(s, a)\right)\right\}-1\right],
$$

where $\vartheta(s, a)$ is defined in (A.2). Then,

$$
\mathrm{E} \exp \left\{\mathrm{i} N^{-1 / \alpha(1+b)} \sum_{i=1}^{N} \sum_{t=1}^{m} \theta_{t} X_{i t}\right\}=\left(1+\frac{\Theta_{N}}{N}\right)^{N} .
$$

Similarly as in the proof of Theorem 2.1 , it suffices to show that

$$
\lim _{N \rightarrow \infty} \Theta_{N}=-K\left|\sum_{t=1}^{m} \theta_{m}\right|^{\alpha(1+b)}, \quad K:=\alpha^{-(b+1)} \psi(1) \int_{0}^{\infty}\left(1-\mathrm{e}^{-z}\right) z^{-(b+2)} \mathrm{d} z .
$$

To prove (A.9), split

$$
\sum_{s \in \mathbb{Z}}|\vartheta(s, a)|^{\alpha} h\left(N^{-1 / \alpha(1+b)} \vartheta(s, a)\right)=\sum_{s \leq 0} \cdots+\sum_{s=1}^{m} \cdots=: \Sigma_{1}+\Sigma_{2} .
$$

Note that $\Sigma_{2}$ is uniformly bounded in $a \in[0,1)$ and $N \geq 1$ and $N^{-1 /(1+b)}=o\left(N^{-1}\right)$ for $b<0$. Therefore, it suffices to prove (A.9) for $\Theta_{N}$ replaced by $\Theta_{N 1}:=N \mathrm{E}\left[\mathrm{e}^{-N^{-1 /(1+b)} \Sigma_{1}}-1\right]$. We have

$$
\begin{aligned}
\Theta_{N 1} & =N \int_{1-\epsilon}^{1}\left(\exp \left\{-N^{-1 /(1+b)} \frac{1}{\alpha(1-a)}\left|\sum_{t=1}^{m} \theta_{t}\right|^{\alpha}\right\}-1\right)(1-a)^{b} \psi(a) \mathrm{d} a+o(1) \\
& =N \int_{0}^{\epsilon}\left(\exp \left\{-\frac{1}{\alpha x N^{1 /(1+b)}}\left|\sum_{t=1}^{m} \theta_{t}\right|^{\alpha}\right\}-1\right) \psi(1-x) x^{b} \mathrm{~d} x+o(1) \\
& =-K_{N}(\theta)\left|\sum_{t=1}^{m} \theta_{t}\right|^{\alpha(1+b)}+o(1)
\end{aligned}
$$

where

$$
K_{N}(\theta):=\alpha^{-(b+1)} \psi(1) \int_{0}^{\infty} \mathbf{1}\left(z>\delta_{N}(\theta)\right)\left(1-\mathrm{e}^{-z}\right) z^{-(b+2)} \mathrm{d} z
$$

and $\delta_{N}(\theta):=(\alpha \epsilon)^{-1} N^{-1 /(1+b)}\left|\sum_{t=1}^{m} \theta_{t}\right|^{\alpha} \rightarrow 0(N \rightarrow \infty)$. Since $\lim _{N \rightarrow \infty} K_{N}(\theta)=K$ by the dominated convergence theorem, this proves (A.9) and the proposition. 
Proof of Proposition 2.4. It suffices to prove the proposition for simple functions $h \in$ $L^{\alpha}(\mathbb{Z} \times(-1,1))$ of the form $h(t, a)=\sum_{i=1}^{n} h_{i t} \mathbf{1}\left(|t| \leq n, a \in A_{i}\right)$, where $A_{i} \subset(-1,1)$, $i=1, \ldots, n$, are disjoint Borel sets. For such $h$,

$$
M(h)=\sum_{|t| \leq n} \sum_{i=1}^{n} h_{i t} M_{t}\left(A_{i}\right)
$$

is a finite sum of $\alpha$-stable RVs. By linearity of both sides of (2.11) in $h$ and independence of $M_{t}\left(A_{i}\right)$ and $M_{s}\left(A_{j}\right), s \neq t$, it suffices to check (2.11) for $h(t, a)=\mathbf{1}(t=s, a \in A)$, or

$$
\mathrm{E}\left[M_{S}(A) \mid M_{S}(-1,1)\right]=\Phi(A) M_{S}(-1,1)
$$

for any Borel set $A \subset(-1,1)$. By standard arguments, (A.10) is equivalent to

$$
\mathrm{E}\left[M_{S}(A) \mathrm{e}^{\mathrm{i} \theta M_{S}(-1,1)}\right]=\Phi(A) \mathrm{E}\left[M_{S}(-1,1) \mathrm{e}^{\mathrm{i} \theta M_{S}(-1,1)}\right], \quad \theta \in \mathbb{R} .
$$

Let $\kappa_{A}(\theta):=\mathrm{E}\left[\mathrm{e}^{\mathrm{i} \theta M_{s}(-1,1)}\right], \kappa(\theta):=\kappa_{(-1,1)}(\theta)$, and $A^{c}:=(-1,1) \backslash A$. Then (A.11) can be rewritten as

$$
\kappa_{A}^{\prime}(\theta) \kappa_{A^{c}}(\theta)=\Phi(A) \kappa^{\prime}(\theta) .
$$

The above equality is immediate from $\kappa_{A}(\theta) \kappa_{A^{c}}(\theta)=\kappa(\theta)$ and $\kappa_{A}(\theta)=(\kappa(\theta))^{\Phi(A)}$ (the last relation follows from the form of the characteristic functional in (2.5) and the fact that $\omega(\theta)$ in (2.2) depends only on the sign of $\theta$ ).

Proof of Theorem 3.1. (i) We will prove the one-dimensional convergence in (3.4) at $\tau=1$ only, since the general case in (3.4) follows analogously. In view of (2.7) and (3.2), it suffices to prove that, for any $\theta \in \mathbb{R}$,

$$
\begin{aligned}
n^{-H \alpha} & \sum_{s \in \mathbb{Z}} \mathrm{E}\left|\sum_{t=1}^{n} a^{t-s} \mathbf{1}(s \leq t)\right|^{\alpha} \omega\left(\theta \sum_{t=1}^{n} a^{t-s} \mathbf{1}(s \leq t)\right) \\
& \rightarrow c \int_{\mathbb{R}} \int_{\mathbb{R}_{+}}|f(x, 1-s)-f(x,-s)|^{\alpha} \omega(\theta(f(x, 1-s)-f(x,-s))) x^{b-\alpha} \mathrm{d} s \mathrm{~d} x .
\end{aligned}
$$

Note that the expressions inside $\omega$ on both sides of (A.12) are positive or negative depending on the sign of $\theta$ and $\omega(\theta)=\omega(\operatorname{sgn}(\theta))$. Therefore, it suffices to show (A.12) for $\theta=1$ alone. To this end, let us denote the left- and right-hand sides of (A.12) (with $\theta=1$ ) by $J_{n}$ and $J$, respectively. Split $J=J_{1}+J_{2}$, where

$$
\begin{aligned}
J_{1} & :=\psi(1) \omega(1) \int_{-\infty}^{0} \mathrm{~d} s \int_{0}^{\infty}|f(x, 1-s)-f(x,-s)|^{\alpha} x^{b-\alpha} \mathrm{d} x \\
& =\psi(1) \omega(1) \alpha^{-1} \int_{0}^{\infty}\left(1-\mathrm{e}^{-y}\right)^{\alpha} y^{b-\alpha-1} \mathrm{~d} y, \\
J_{2} & :=\psi(1) \omega(1) \int_{0}^{1} \mathrm{~d} s \int_{0}^{\infty}|f(x, 1-s)|^{\alpha} x^{b-\alpha} \mathrm{d} x \\
& =\psi(1) \omega(1) \int_{0}^{1} \mathrm{~d} u \int_{0}^{\infty}\left(1-\mathrm{e}^{-x(1-u)}\right)^{\alpha} x^{b-\alpha} \mathrm{d} x,
\end{aligned}
$$


according to the definition of $f$ in (1.5). Next, write $J_{n}=J_{n 1}+J_{n 2}$, where

$$
\begin{aligned}
J_{n 1} & :=n^{-H \alpha} \omega(1) \sum_{s=-\infty}^{0} \int_{0}^{1}\left|\sum_{t=1}^{n} a^{t-s}\right|^{\alpha}(1-a)^{b} \psi(a) \mathrm{d} a \\
& =n^{-H \alpha} \omega(1) \int_{0}^{1} \frac{1}{1-a^{\alpha}}\left|\frac{a\left(1-a^{n}\right)}{1-a}\right|^{\alpha}(1-a)^{b} \psi(a) \mathrm{d} a \\
& =\omega(1) \int_{0}^{\infty} \frac{(1-y / n)^{\alpha}}{n\left(1-(1-y / n)^{\alpha}\right)}\left(1-\left(1-\frac{y}{n}\right)^{n}\right)^{\alpha} y^{b-\alpha} \psi\left(1-\frac{y}{n}\right) \\
& \rightarrow \frac{\psi(1) \omega(1)}{\alpha} \int_{0}^{\infty}\left(1-\mathrm{e}^{-y}\right)^{\alpha} y^{b-\alpha-1} \mathrm{~d} y \\
& =J_{1}
\end{aligned}
$$

by the dominated convergence theorem as $n \rightarrow \infty$. In a similar way,

$$
\begin{aligned}
J_{n 2} & :=n^{-H \alpha} \omega(1) \sum_{s=1}^{n} \int_{0}^{1}\left|\sum_{t=1}^{n} a^{t-s} \mathbf{1}(s \leq t)\right|^{\alpha}(1-a)^{b} \psi(a) \mathrm{d} a \\
& =n^{-H \alpha} \omega(1) \int_{0}^{1} \sum_{s=1}^{n}\left|\frac{1-a^{n-s+1}}{1-a}\right|^{\alpha}(1-a)^{b} \psi(a) \mathrm{d} a \\
& =\omega(1) \int_{0}^{\infty} \frac{1}{n} \sum_{s=1}^{n}\left(1-\left(1-\frac{y}{n}\right)^{n-s+1}\right)^{\alpha} y^{b-\alpha} \psi\left(1-\frac{y}{n}\right) \mathbf{1}(0<y<\epsilon n) \mathrm{d} y+o(1) \\
& \rightarrow \psi(1) \omega(1) \int_{0}^{\infty} \int_{0}^{1}\left(1-\mathrm{e}^{-y(1-u)}\right)^{\alpha} y^{b-\alpha} \mathrm{d} y \mathrm{~d} u \\
& =J_{2} .
\end{aligned}
$$

This proves part (i).

(ii) Denote by $\left\{L_{n}(\tau), \tau\right\}$ the process on the left-hand side of (3.5). It suffices to prove that, for any $m \geq 1$ and any $0=: \tau_{0}<\tau_{1}<\cdots<\tau_{m}, \theta_{1} \in \mathbb{R}, \ldots, \theta_{m} \in \mathbb{R}$,

$$
\sum_{k=1}^{m} \theta_{k}\left(L_{n}\left(\tau_{k}\right)-L_{n}\left(\tau_{k-1}\right)\right) \stackrel{\mathrm{D}}{\rightarrow} \sum_{k=1}^{m} \theta_{k}\left(L\left(\tau_{k}\right)-L\left(\tau_{k-1}\right)\right) .
$$

Rewrite $L_{n}\left(\tau_{k}\right)-L_{n}\left(\tau_{k-1}\right)=\Delta L_{n}^{\prime}\left(\tau_{k}\right)+\Delta L_{n}^{\prime \prime}\left(\tau_{k}\right)$, where

$$
\begin{aligned}
& \Delta L_{n}^{\prime}\left(\tau_{k}\right):=n^{-1 / \alpha} \sum_{\left[n \tau_{k-1}\right]<s \leq\left[n \tau_{k}\right]} \sum_{s \leq t \leq\left[n \tau_{k}\right]} \int_{0}^{1} a^{t-s} M_{s}(\mathrm{~d} a), \\
& \Delta L_{n}^{\prime \prime}\left(\tau_{k}\right):=n^{-1 / \alpha} \sum_{s \leq\left[n \tau_{k-1}\right]\left[n \tau_{k-1}\right]<t \leq\left[n \tau_{k}\right]} \int_{0}^{1} a^{t-s} M_{s}(\mathrm{~d} a) .
\end{aligned}
$$

Since $\Delta L_{n}^{\prime}\left(\tau_{k}\right), k=1, \ldots, m$, are independent, it suffices to prove that, for any $k=1, \ldots, m$,

$$
\Delta L_{n}^{\prime}\left(\tau_{k}\right) \stackrel{\mathrm{D}}{\rightarrow} L\left(\tau_{k}\right)-L\left(\tau_{k-1}\right), \quad \Delta L_{n}^{\prime \prime}\left(\tau_{k}\right)=o_{p}(1) .
$$


Moreover, it suffices to prove the last relations for $k=1$ and $\tau_{k}=1$ only; in other words, to prove that, for any $\theta \in \mathbb{R}$,

$$
\begin{aligned}
& n^{-1} \sum_{s=1}^{n} \mathrm{E}\left(\sum_{t=s}^{n} a^{t-s}\right)^{\alpha} \omega\left(\theta \sum_{t=s}^{n} a^{t-s}\right) \rightarrow K \omega(\theta), \\
& n^{-1} \sum_{s \leq 0} \mathrm{E}\left(\sum_{t=1}^{n} a^{t-s}\right)^{\alpha} \omega\left(\theta \sum_{t=1}^{n} a^{t-s}\right) \rightarrow 0 .
\end{aligned}
$$

Similarly as in the proof of Proposition 3.4, it suffices to prove the above relations for $\omega(\theta) \equiv 1$, viz.

$$
J_{n 1}:=n^{-1} \sum_{s \leq 0} \mathrm{E}\left(\sum_{t=1}^{n} a^{t-s}\right)^{\alpha} \rightarrow 0, \quad J_{n 2}:=n^{-1} \sum_{s=1}^{n} \mathrm{E}\left(\sum_{t=s}^{n} a^{t-s}\right)^{\alpha} \rightarrow K .
$$

Consider

$$
J_{n 1}=n^{-1} \int_{0}^{1} \frac{(1-x)^{\alpha}}{1-(1-x)^{\alpha}}\left(1-(1-x)^{n}\right)^{\alpha} x^{b-\alpha} \psi(1-x) \mathrm{d} x
$$

If $b>\alpha$ then, clearly, $J_{n 1} \leq C n^{-1} \int_{0}^{1} x^{b-\alpha-1} \psi(1-x) \mathrm{d} x=O\left(n^{-1}\right)$ since the last integral converges. Let $0<b<\alpha$. Then, for any $\epsilon>0$, similarly as above

$$
J_{n 1}=\frac{1}{n^{b-\alpha+1}} \int_{0}^{\epsilon n} \frac{(1-y / n)^{\alpha}}{n\left(1-(1-y / n)^{\alpha}\right)}\left(1-\left(1-\frac{y}{n}\right)^{n}\right)^{\alpha} y^{b-\alpha} \psi\left(1-\frac{y}{n}\right) \mathrm{d} y+O\left(\frac{1}{n}\right)
$$

where the last integral tends to $\psi(1) \alpha^{-1} \int_{0}^{\infty}\left(1-\mathrm{e}^{-y}\right)^{\alpha} y^{b-\alpha-1} \mathrm{~d} y<\infty$, implying that $J_{n 2}=$ $O\left(1 / n^{b-\alpha+1}\right)=o(1)$. For $b=\alpha$, a similar argument yields $J_{n 2}=O\left(n^{-1} \log n\right)=o(1)$. This proves the first convergence in (A.13).

Next, by the dominated convergence theorem,

$$
J_{n 2}=n^{-1} \sum_{k=0}^{n-1} \int_{0}^{1} x^{b-\alpha}\left(1-(1-x)^{k}\right)^{\alpha} \psi(1-x) \mathrm{d} x \rightarrow \int_{0}^{1} x^{b-\alpha} \psi(1-x) \mathrm{d} x=K,
$$

proving the second relation in (A.13) and the theorem.

Proof of Theorem 3.2. (i) In view of Theorem 3.1(i), it suffices to show that $n^{-2 H} \sum_{t=1}^{n} \bar{X}_{t}^{2}=$ $o_{p}(1)$, with $H$ as in (3.3). The last relation follows from $H>1 / \alpha$ and Loève (1963, p. 387). See also Heyde and Yang (1997, proof of Theorem 1). This proves part (i).

(ii) According to Theorem 3.1(ii), it suffices to show that $D_{n}^{-1}$ is bounded in probability, where $D_{n}:=n^{-2 / \alpha} \sum_{t=1}^{n} \bar{X}_{t}^{2}$. Decompose $D_{n}=\sum_{i=1}^{3} D_{n i}$, where $D_{n i}$ are defined in (A.14), below. Then $D_{n}^{-1}=O_{p}(1)$ follows from the following three facts: (d1) $D_{n 1}=$ $o_{p}(1)$, (d2) $D_{n 2} \geq 0$ a.s., and (d3) $D_{n 3} \stackrel{\mathrm{D}}{\rightarrow} Z$, where $Z>0$ a.s. To this end, let $\bar{X}_{t}=$ $\sum_{s \leq t} U_{t, s}, U_{t, s}:=\int_{0}^{1} a^{t-s} M_{s}(\mathrm{~d} a) \mathbf{1}(s \leq t)$, and

$$
\begin{gathered}
D_{n 1}:=n^{-2 / \alpha} \sum_{t=1}^{n} \sum_{s_{1} \neq s_{2}} U_{t, s_{1}} U_{t, s_{2}}, \\
D_{n 2}:=n^{-2 / \alpha} \sum_{t=1}^{n} \sum_{s \neq t} U_{t, s}^{2}, \quad D_{n 3}:=n^{-2 / \alpha} \sum_{t=1}^{n} U_{t, t}^{2} .
\end{gathered}
$$

Fact (d2) is obvious. Fact (d3) holds since $U_{t, t}, t=1, \ldots, n$, are i.i.d. $\alpha$-stable RVs, so that $U_{t, t}^{2} \in D(\alpha / 2)$ and $D_{n 3} \stackrel{\mathrm{D}}{\rightarrow} Z$, where $Z$ is a strictly positive $\alpha / 2$-stable RV. 
Let us prove (d1). Write $D_{n 1}=\sum_{s<n} \Gamma_{n, s}$, where $\Gamma_{n, s}:=2 n^{-2 / \alpha} \sum_{t=1}^{n} \sum_{v<s} U_{t, s} U_{t, v}$. Let $\mathcal{F}_{s}$ be the $\sigma$-algebra generated by RVs $M_{v}(A), v \leq s, A \subset(-1,1)$. Then $\left\{\Gamma_{n, s}, \mathcal{F}_{s}\right.$, $s \in \mathbb{Z}\}$ is a martingale difference sequence. Hence, for any $1<r<\alpha$, we have $\mathrm{E}\left|D_{n 1}\right|^{r} \leq$ $2 \sum_{s \leq n} \mathrm{E}\left|\Gamma_{n, s}\right|^{r}$. By a similar backward martingale property,

$$
\mathrm{E}\left|\Gamma_{n, s}\right|^{r} \leq 2 \sum_{v<s} n^{-2 r / \alpha} \mathrm{E}\left|\sum_{t=1}^{n} U_{t, s} U_{t, v}\right|^{r} .
$$

Hence, using the independence of $U_{t, s}$ and $U_{t, v}, v<s$, and Hölder's inequality, for any $1<r<\alpha$, we obtain

$$
\begin{aligned}
\mathrm{E}\left|D_{n 1}\right|^{r} & \leq 4 n^{-2 r / \alpha} \sum_{v<s \leq n} \mathrm{E}\left(\sum_{t=1}^{n} U_{t, s} U_{t, v}\right)^{r} \\
& \leq 4 n^{-2 r / \alpha} n^{r-1} \sum_{v<s \leq n} \sum_{t=1}^{n} \mathrm{E}\left|U_{t, s}\right|^{r} \mathrm{E}\left|U_{t, v}\right|^{r} \\
& \leq 4 n^{-2 r / \alpha} n^{r} Q_{r},
\end{aligned}
$$

where $Q_{r}:=\left(\sum_{s \geq 0} \mathrm{E}\left|U_{s, 0}\right|^{r}\right)^{2}$. Since $r-2 r / \alpha<0$, for (d1), it suffices to show that $Q_{r}<\infty$. From Samorodnitsky and Taqqu (1994, Property 1.2.17) we have $\mathrm{E}\left|U_{s, 0}\right|^{r} \leq C\left(\mathrm{E}\left|a^{s}\right|^{\alpha}\right)^{r / \alpha}$, where $\mathrm{E}\left|a^{s}\right|^{\alpha} \leq C \int_{0}^{1} x^{b}(1-x)^{s \alpha} \mathrm{d} x \leq C s^{-1-b}$ and, therefore, $Q_{r}<\infty$ for $\alpha /(1+b)<$ $r<\alpha$. This completes the proof.

Proof of Theorem 3.3. From (2.7) and the definition of the codifference for $t \geq 1$, we obtain

$$
\operatorname{cod}\left(\bar{X}_{0}, \bar{X}_{t}\right)=\operatorname{Re}(\omega(1)) \Lambda_{1}(t)-\mathrm{i} \operatorname{Im}(\omega(1)) \Lambda_{2}(t),
$$

where $\Lambda_{i}(t):=\mathrm{E} R_{i}, i=1,2$, and

$$
\begin{aligned}
& R_{1}:=\frac{1-\left|1-a^{t}\right|^{\alpha}+\left|a^{t}\right|^{\alpha}}{1-|a|^{\alpha}}, \\
& R_{2}:=\sum_{s \leq 0}\left|a^{t-s}-a^{-s}\right|^{\alpha} \operatorname{sgn}\left(a^{t-s}-a^{-s}\right)+\sum_{s=1}^{t}\left|a^{t-s}\right|^{\alpha} \operatorname{sgn}\left(a^{t-s}\right) .
\end{aligned}
$$

Next, decompose $\Lambda_{i}(t)=\sum_{j=1}^{4} \Lambda_{i j}(t)$, where

$$
\begin{array}{ll}
\Lambda_{i 1}(t):=\mathrm{E} R_{i} \mathbf{1}(1-\epsilon<a<1), & \Lambda_{i 3}(t):=\mathrm{E} R_{i} \mathbf{1}(0<a<1-\epsilon), \\
\Lambda_{i 2}(t):=\mathrm{E} R_{i} \mathbf{1}(-1<a<-1+\epsilon), & \Lambda_{i 4}(t):=\mathrm{E} R_{i} \mathbf{1}(-1+\epsilon<a<0),
\end{array}
$$

and $\epsilon>0$ is a small number. It is easy to check that, for any $\epsilon>0$,

$$
\Lambda_{i j}(t)=O\left(\mathrm{e}^{-\tilde{c} t}\right)=o\left(t^{-b_{1} \vee b_{2}}\right), \quad i=1,2, j=3,4, \text { there exists } \tilde{c}>0,
$$

decay exponentially and, hence, are negligible in (3.8). Consider the terms $\Lambda_{i j}(t), i, j=1,2$. We have

$$
\begin{aligned}
\Lambda_{11}(t) & =\int_{1-\epsilon}^{1} \frac{1-\left|1-a^{t}\right|^{\alpha}+\left|a^{t}\right|^{\alpha}}{1-|a|^{\alpha}}(1-a)^{b_{1}} \psi(a) \mathrm{d} a \\
& =\int_{0}^{\epsilon} \frac{1-\left(1-(1-x)^{t}\right)^{\alpha}+(1-x)^{t \alpha}}{1-(1-x)^{\alpha}} x^{b_{1}} \psi(1-x) \mathrm{d} x \\
& =C_{11}(t) t^{-b_{1}},
\end{aligned}
$$


where

$$
\begin{aligned}
C_{11}(t):= & \psi(1) \alpha^{-1} \int_{0}^{\infty} f(t, y)\left(1-\left(1-\mathrm{e}^{-y}\right)^{\alpha}+\mathrm{e}^{-y \alpha}\right) y^{b_{1}-1} \mathrm{~d} y, \\
f(t, y):= & \frac{1-\left(1-(1-y / t)^{t}\right)^{\alpha}+(1-y / t)^{t \alpha}}{1-\left(1-\mathrm{e}^{-y}\right)^{\alpha}+\mathrm{e}^{-y \alpha}} \frac{\alpha(y / t)}{1-(1-y / t)^{\alpha}} \frac{\psi(1-y / t)}{\psi(1)} \\
& \times \mathbf{1}(0<y<\epsilon t) .
\end{aligned}
$$

Observe that $f(t, y) \rightarrow 1(t \rightarrow \infty)$ for any $y \in(0, \infty)$, and, moreover, $|f|$ is bounded in $y \in(0, \infty)$ uniformly in $t \rightarrow \infty$. Hence, by the dominated convergence theorem,

$$
C_{11}(t)=\psi(1) \alpha^{-1} \int_{0}^{\infty}\left(1-\left(1-\mathrm{e}^{-y}\right)^{\alpha}+\mathrm{e}^{-y \alpha}\right) y^{b_{1}-1} \mathrm{~d} y+o(1) .
$$

In a similar way,

$$
\Lambda_{12}(t)=\int_{0}^{\epsilon} \frac{1-\left(1-(-1)^{t}(1-x)^{t}\right)^{\alpha}+(1-x)^{t \alpha}}{1-(1-x)^{\alpha}} x^{b_{2}} \psi(x-1) \mathrm{d} x=C_{12}(t) t^{-b_{2}},
$$

where

$$
C_{12}(t)=\psi(-1) \alpha^{-1} \int_{0}^{\infty}\left[\mathrm{e}^{-y \alpha}+1-\left(1-(-1)^{t} \mathrm{e}^{-y}\right)^{\alpha}\right] y^{b_{2}-1} \mathrm{~d} y+o(1) .
$$

Next, using $\operatorname{sgn}\left(a^{t-s}\right)=\operatorname{sgn}\left(a^{t}\right) \operatorname{sgn}\left(a^{-s}\right)$ and

$$
\begin{gathered}
\operatorname{sgn}\left(a^{t-s}-a^{-s}\right)=-1, \quad \operatorname{sgn}\left(a^{t-s}\right)=+1, \quad \text { for } a>0, \\
\operatorname{sgn}\left(a^{t-s}-a^{-s}\right)=-\left((-1)^{-s}\right), \quad \operatorname{sgn}\left(a^{t-s}\right)=(-1)^{t}\left((-1)^{-s}\right), \quad \text { for } a<0,
\end{gathered}
$$

we can rewrite

$$
R_{2}=\frac{1-\left(1-a^{t}\right)^{\alpha}-a^{t \alpha}}{1-a^{\alpha}} \mathbf{1}(a>0)+\frac{1-\left(1-a^{t}\right)^{\alpha}-(-1)^{t}\left|a^{t}\right|^{\alpha}}{1+|a|^{\alpha}} \mathbf{1}(a<0) .
$$

Whence, similarly as above,

$$
\Lambda_{21}(t)=\int_{0}^{\epsilon} \frac{1-\left(1-(1-x)^{t}\right)^{\alpha}-(1-x)^{t \alpha}}{1-(1-x)^{\alpha}} x^{b_{1}} \psi(1-x) \mathrm{d} x=C_{21}(t) t^{-b_{1}},
$$

where

$$
C_{21}(t)=\psi(1) \alpha^{-1} \int_{0}^{\infty}\left(1-\left(1-\mathrm{e}^{-y}\right)^{\alpha}-\mathrm{e}^{-y \alpha}\right) y^{b_{1}-1} \mathrm{~d} y+o(1) .
$$

Finally,

$$
\begin{aligned}
\Lambda_{22}(t) & =\int_{0}^{\epsilon} \frac{1-\left(1-(-1)^{t}(1-x)^{t}\right)^{\alpha}-(-1)^{t}(1-x)^{t \alpha}}{1+(1-x)^{\alpha}} x^{b_{2}} \psi(x-1) \mathrm{d} x \\
& =C_{22}(t) t^{-b_{2}-1} \\
& =o\left(t^{-b_{2}}\right),
\end{aligned}
$$

where $C_{22}(t)=\psi(-1) 2^{-1} \int_{0}^{\infty}\left(1-\left(1-(-1)^{t} \mathrm{e}^{-y}\right)^{\alpha}-\mathrm{e}^{-y \alpha}\right) y^{b_{2}} \mathrm{~d} y+o(1)$.

The asymptotics in (3.8) follow from (A.15) and (A.16)-(A.23).

\section{Acknowledgements}

We thank the anonymous referee for useful comments. This research was supported by the Lithuanian State Science and Studies Foundation, grant number T-70/09. 


\section{References}

AstrausKas, A. (1983). Limit theorems for sums of linearly generated random variables. Lithuanian Math. J. 23, $127-134$

AstrausKas, A., LeVy, J. B. And TAQQU, M. S. (1991). The asymptotic dependence structure of the linear fractional Lévy motion. Lithuanian Math. J. 31, 1-19.

Brandt, A. (1986). The stochastic equation $Y_{n+1}=A_{n} Y_{n}+B_{n}$ with stationary coefficients. Adv. Appl. Prob. 18, 211-220.

Celov, D., Leipus, R. And PhilipPe, A. (2007). Time series aggregation, disaggregation, and long memory. Lithuanian Math. J. 47, 379-393.

CioczeK-Georges, R. and Mandelbrot, B. B. (1995). Stable fractal sums of pulses: the general case. Preprint. Available at http://yale.academia.edu/BenoitMandelbrot/Papers/21711.

Cioczek-Georges, R., Mandelbrot, B. B., Samorodnitsky, G. and Taqqu, M. S. (1995). Stable fractal sums of pulses: the cylindrical case. Bernoulli 1, 201-216.

Cox, D. R. (1984). Long-range dependence: a review. In Statistics: An Appraisal, eds H. A. David and H. T. David. Iowa State University Press, Iowa, pp. 55-74.

Ding, Z. AND GRANGER, C. W. J. (1996). Modeling volatility persistence of speculative returns: a new approach. J. Econometrics 73, 185-215.

Feller, W. (1971). An Introduction to Probability Theory and Its Applications, Vol. II, 2nd edn. John Wiley, New York.

Giraitis, L., Leipus, R. AND Surgailis, D. (2010). Aggregation of random coefficient GLARCH(1,1) process. Econometric Theory 26, 406-425.

GonÇalves, E. And Gouriéroux, C. (1988). Aggrégation de processus autorégressifs d'ordre 1. Ann. Econom. Statist. 12, 127-149.

Granger, C. W. J. (1980). Long memory relationship and the aggregation of dynamic models. J. Econometrics 14, $227-238$

Heyde, C. C. AND Yang, Y. (1997). On defining long-range dependence. J. Appl. Prob. 34, 939-944.

Ibragimov, I. A. And LinniK, Y. V. (1971). Independent and Stationary Sequences of Random Variables. WoltersNoordhoff, Groningen.

KAZAKevičIUS, V., LeIPUS, R. AND VIANO, M.-C. (2004). Stability of random coefficient ARCH models and aggregation schemes. J. Econometrics 120, 139-158.

Koul, H. L. And Surgailis, D. (2001). Asymptotics of empirical processes of long memory moving averages with infinite variance. Stoch. Process. Appl. 91, 309-336.

Lamperti, J. (1962). Semi-stable stochastic processes. Trans. Amer. Math. Soc. 104, 62-78.

LeIPUS, R. AND VIANO, M.-C. (2002). Aggregation in ARCH models. Lithuanian Math. J. 42, 54-70.

LoÈve, M. (1963). Probability Theory, 3rd edn. Van Nostrand, Princeton, NJ.

Mıкоsсн, T. (2003). Modelling dependence and tails of financial time series. In Extreme Values in Finance, Telecommunications and the Environment, eds B. Finkenstädt and H. Rootzén, Chapman and Hall, New York, pp. 185-286.

Mikosch. T. AND SAmorodnitsky, G. (2000). Ruin probability with claims modeled by a stationary ergodic stable process. Ann. Prob. 28, 1814-1851.

OpPenheim, G. AND Viano, M.-C. (2004). Aggregation of random parameters Ornstein-Uhlenbeck or AR processes: some convergence results. J. Time Ser. Anal. 25, 335-350.

Puplinskaité, D. AND Surgailis, D. (2009). Aggregation of random-coefficient AR(1) process with infinite variance and common innovations. Lithuanian Math. J. 49, 446-463.

Robinson, P. M. (1978). Statistical inference for a random coefficient autoregressive model. Scand. J. Statist. 5, 163-168.

RosińsKi, J. (1995). On the structure of stationary stable processes. Ann. Prob. 23, 1163-1187.

SAmorodnitsky, G. (2004). Extreme value theory, ergodic theory and the boundary between short memory and long memory for stationary stable processes. Ann. Prob. 32, 1438-1468.

SAmorodnitsky, G. ANd TAqQu, M. S. (1994). Stable Non-Gaussian Random Processes. Chapman and Hall, New York.

SurgaILIS, D. (1979). On the Markov property of a class of linear infinitely divisible fields. Z. Wahrscheinlichkeitsth 49, 293-311.

Surgailis, D., Rosiński, J., Mandrekar, V. And CAmbanis, S. (1992). Stable generalized moving averages. Preprint, University of North Carolina.

Surgailis, D., Rosiński, J., Mandrekar, V. and Cambanis, S. (1993). Stable mixed moving averages. Prob. Theory Relat. Fields 97, 543-558.

ZafFAroni, P. (2004). Contemporaneous aggregation of linear dynamic models in large economies. J. Econometrics 120, 75-102.

ZafFaroni, P. (2007a). Aggregation and memory of models of changing volatility. J. Econometrics 136, 237-249.

Zaffaroni, P. (2007b). Contemporaneous aggregation of GARCH processes. J. Time Ser. Anal. 28, 521-544. 